\title{
Discovery of a brown dwarf companion to the star HIP $64892^{\star}$
}

\author{
A. Cheetham ${ }^{1}$, M. Bonnefoy ${ }^{2}$, S. Desidera ${ }^{3}$, M. Langlois ${ }^{4,5}$, A. Vigan ${ }^{5}$, T. Schmidt ${ }^{6}$, J. Olofsson ${ }^{7,8,9}$, \\ G. Chauvin ${ }^{2,8}$, H. Klahr ${ }^{7}$, R. Gratton ${ }^{3}$, V. D'Orazi ${ }^{3}$, T. Henning ${ }^{7}$, M. Janson ${ }^{7,10}$, B. Biller ${ }^{7}, 11$,

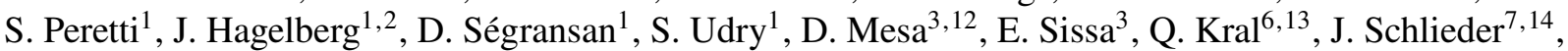 \\ A.-L. Maire ${ }^{7}$, C. Mordasini ${ }^{7}, 15$, F. Menard ${ }^{2}$, A. Zurlo ${ }^{5,16}$, J.-L. Beuzit ${ }^{2}$, M. Feldt ${ }^{7}$, D. Mouillet ${ }^{2}$, M. Meyer ${ }^{17,18}$, \\ A.-M. Lagrange ${ }^{2}$, A. Boccaletti ${ }^{6}$, M. Keppler ${ }^{7}$, T. Kopytova ${ }^{7,19,20}$, R. Ligi ${ }^{5}$, D. Rouan ${ }^{6}$, H. Le Coroller ${ }^{5}$,

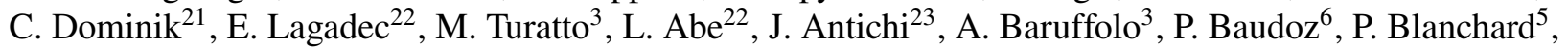 \\ T. Buey ${ }^{6}$, M. Carbillet ${ }^{22}$, M. Carle ${ }^{5}$, E. Cascone ${ }^{24}$, R. Claudi ${ }^{3}$, A. Costille ${ }^{5}$, A. Delboulbé ${ }^{2}$, V. De Caprio ${ }^{24}$, \\ K. Dohlen ${ }^{5}$, D. Fantinel ${ }^{3}$, P. Feautrier ${ }^{2}$, T. Fusco ${ }^{25}$, E. Giro ${ }^{3}$, L. Gluck ${ }^{2}$, N. Hubin ${ }^{26}$, E. Hugot $^{5}$, M. Jaquet ${ }^{5}$, \\ M. Kasper ${ }^{26}$, M. Llored ${ }^{5}$, F. Madec ${ }^{5}$, Y. Magnard ${ }^{2}$, P. Martinez ${ }^{22}$, D. Maurel ${ }^{2}$, D. Le Mignant ${ }^{5}$, \\ O. Möller-Nilsson ${ }^{7}$, T. Moulin ${ }^{2}$, A. Origné ${ }^{5}$, A. Pavlov ${ }^{7}$, D. Perret ${ }^{6}$, C. Petit ${ }^{25}$, J. Pragt ${ }^{27}$, P. Puget ${ }^{2}$, \\ P. Rabou ${ }^{2}$, J. Ramos ${ }^{7}$, F. Rigal ${ }^{21}$, S. Rochat ${ }^{2}$, R. Roelfsema ${ }^{27}$, G. Rousset ${ }^{6}$, A. Roux ${ }^{2}$, B. Salasnich ${ }^{3}$, \\ J.-F. Sauvage ${ }^{25}$, A. Sevin ${ }^{6}$, C. Soenke ${ }^{26}$, E. Stadler ${ }^{2}$, M. Suarez ${ }^{28}$, L. Weber ${ }^{1}$, and F. Wildi ${ }^{1}$
}

(Affiliations can be found after the references)

Received 16 January 2018 / Accepted 6 March 2018

\begin{abstract}
We report the discovery of a bright, brown dwarf companion to the star HIP 64892, imaged with VLT/SPHERE during the SHINE exoplanet survey. The host is a B9.5V member of the Lower-Centaurus-Crux subgroup of the Scorpius Centaurus OB association. The measured angular separation of the companion $(1.2705 \pm 0.0023$ ”) corresponds to a projected distance of $159 \pm 12$ AU. We observed the target with the dual-band imaging and long-slit spectroscopy modes of the IRDIS imager to obtain its spectral energy distribution (SED) and astrometry. In addition, we reprocessed archival NACO $L$-band data, from which we also recover the companion. Its SED is consistent with a young ( $<30 \mathrm{Myr}$ ), low surface gravity object with a spectral type of $\mathrm{M} 9_{\gamma} \pm 1$. From comparison with the BT-Settl atmospheric models we estimate an effective temperature of $T_{\text {eff }}=2600 \pm 100 \mathrm{~K}$, and comparison of the companion photometry to the COND evolutionary models yields a mass of $\sim 29-37 M_{\mathrm{J}}$ at the estimated age of $16_{-7}^{+15}$ Myr for the system. The star HIP 64892 is a rare example of an extreme-mass ratio system $(q \sim 0.01)$ and will be useful for testing models relating to the formation and evolution of such low-mass objects.
\end{abstract}

Key words. brown dwarfs - stars: individual: HIP 64892 - techniques: high angular resolution - planets and satellites: detection planets and satellites: atmospheres

\section{Introduction}

While evidence suggests that the frequency of short period stellar and planetary-mass companions to main sequence stars is high, there appears to be a relative lack of companions in the brown dwarf regime (Grether \& Lineweaver 2006). These objects appear to exist in an overlap region of formation processes, as the low-mass tail of stellar binary formation and as the high-mass end of the planetary distribution. Observations of brown dwarf companions to young stars then present an important opportunity to study these different formation pathways.

The processes that form companions of all masses appear to have a sensitive dependence on the host star mass, with evidence suggesting that high and intermediate mass stars have more of such companions than their lower mass counterparts (e.g. Bowler et al. 2010; Johnson et al. 2010; Janson et al. 2013; Jones et al. 2014; Bowler et al. 2015; Lannier et al. 2016). However, our

\footnotetext{
* Based on observations collected at the European Organisation for Astronomical Research in the Southern Hemisphere under ESO programmes 096.C-0241 and 198.C-0209 (PI: J.-L. Beuzit), 098.A9007(A) (PI: P. Sarkis), and 087.C-0790(A) (PI: M. Ireland).
}

knowledge of such companions is limited by the small number of objects detected to date, covering a wide range of parameter space in terms of companion mass, primary mass, age, and orbital semi-major axis. Only a few wide-separation objects have been detected around stars with masses $>2 M_{\odot}$, such as HIP 78530B (Lafrenière et al. 2011), $\kappa$ And b (Carson et al. 2013), HR 3549B (Mawet et al. 2015), and HIP 77900B (Aller et al. 2013).

A new generation of dedicated planet-finding instruments such as the Spectro-Polarimetric High-contrast Exoplanet REsearch (SPHERE) instrument (Beuzit et al. 2008) and the Gemini Planet Imager (Macintosh et al. 2008) offer significant improvements in the detection capability for substellar companions, as well as for spectroscopic and astrometric follow-up. The SHINE (SpHere INfrared survey for Exoplanets) survey (Chauvin et al. 2017) utilises the SPHERE instrument at the Very Large Telescope (VLT) to search the close environments of 600 young, nearby stars for substellar and planetary companions. In this paper, we present the imaging discovery and followup spectroscopy of a young, low-mass brown dwarf companion identified during the course of this survey. 
Table 1. Stellar parameters of HIP 64892.

\begin{tabular}{lcl}
\hline \hline Parameter & Value & Ref. \\
\hline V (mag) & $6.799 \pm 0.008$ & Slawson et al. (1992) \\
U-B (mag) & $-0.094 \pm 0.011$ & Slawson et al. (1992) \\
B-V (mag) & $-0.018 \pm 0.006$ & Slawson et al. (1992) \\
V-I (mag) & 0.00 & HIPPARCOS \\
J (mag) & $6.809 \pm 0.023$ & Cutri et al. (2003) \\
H (mag) & $6.879 \pm 0.034$ & Cutri et al. (2003) \\
K (mag) & $6.832 \pm 0.018$ & Cutri et al. (2003) \\
E $(\mathrm{B}-\mathrm{V})$ & $0.01_{--0.01}^{+0.02}$ & This paper \\
Parallax (mas) & $7.98 \pm 0.55$ & van Leeuwen (2007) \\
Distance $(\mathrm{pc})$ & $125 \pm 9 \mathrm{pc}$ & From parallax \\
$\mu_{\alpha}\left(\mathrm{mas} \mathrm{yr}^{-1}\right)$ & $-30.83 \pm 0.50$ & van Leeuwen (2007) \\
$\mu_{\delta}\left(\mathrm{mas} \mathrm{yr}^{-1}\right)$ & $-20.22 \pm 0.43$ & van Leeuwen (2007) \\
$\mathrm{RV}\left(\mathrm{km} \mathrm{s}^{-1}\right)$ & 14.9 & This paper \\
SpT & $\mathrm{B} 9.5 \mathrm{~V}$ & Houk (1993) \\
$T_{\text {eff }}(\mathrm{K})$ & 10400 & SpT+Pecaut calib. \\
$v \sin i\left(\mathrm{~km} \mathrm{~s}^{-1}\right)$ & 178 & This paper \\
Age $(\mathrm{Myr})$ & $16_{-7}^{+15}$ & This paper \\
$M_{\text {star }}\left(M_{\odot}\right)$ & $2.35 \pm 0.09$ & This paper \\
$R_{\text {star }}\left(R_{\odot}\right)$ & $1.79 \pm 0.10 R_{\odot}$, & This paper \\
\hline
\end{tabular}

\section{Stellar properties}

The star HIP 64892 was assigned a B9.5 spectral type by Houk (1993) and classified as a member of the Lower Centaurus Crux (hereafter LCC) association by de Zeeuw et al. (1999) and Rizzuto et al. (2011), with membership probabilities of $99 \%$ and $74 \%$, respectively. A recent update to the BANYAN tool by Gagné et al. (2018) gives a membership probability of $64 \%$ to the LCC subgroup, 33\% to the younger Upper Centaurus Lupus subgroup, and a 3\% probability of HIP 64892 being a field star. Since the star is not included in Gaia Data Release 1, we adopt the trigonometric parallax by van Leeuwen (2007), yielding a distance of $125 \pm 9 \mathrm{pc}$. Photometry of the system is collected in Table 1 . No significant variability is reported by HIPPARCOS (photometric scatter $0.007 \mathrm{mag}$ ).

To further characterise the system, the star was observed with the FEROS spectrograph at the $2.2 \mathrm{~m} \mathrm{MPG}$ telescope operated by the Max Planck Institute for Astronomy. Data were obtained on 2017-Mar-30 and were reduced with the CERES package (Brahm et al. 2017) ${ }^{1}$. A summary of the stellar properties derived from this analysis, and those compiled from the literature, is given in Table 1. From the FEROS spectrum, we measured a radial velocity (RV) of $14.9 \mathrm{~km} \mathrm{~s}^{-1}$ and a projected rotational velocity of $178 \mathrm{~km} \mathrm{~s}^{-1}$, using the cross-correlation function (CCF) procedure described in Chauvin et al. (2017). This latter value is not unusual among stars of similar spectral type, unless significant projection effects are at work.

The FEROS CCF does not show any indication of additional components. This agrees with the conclusions of Chini et al. (2012), who found no evidence of binarity from five RV measurements. The observed RV values from that study were not published, preventing an assessment of possible long-term RV variability ${ }^{2}$. The SPHERE data also do not provide any indication of the presence of bright stellar companions, ruling

\footnotetext{
1 https://github.com/rabrahm/ceres

2 The RV provided by SIMBAD originates from the study of Madsen et al. (2002) and was not derived from spectroscopic measurements but rather from the velocity expected from kinematics.
}

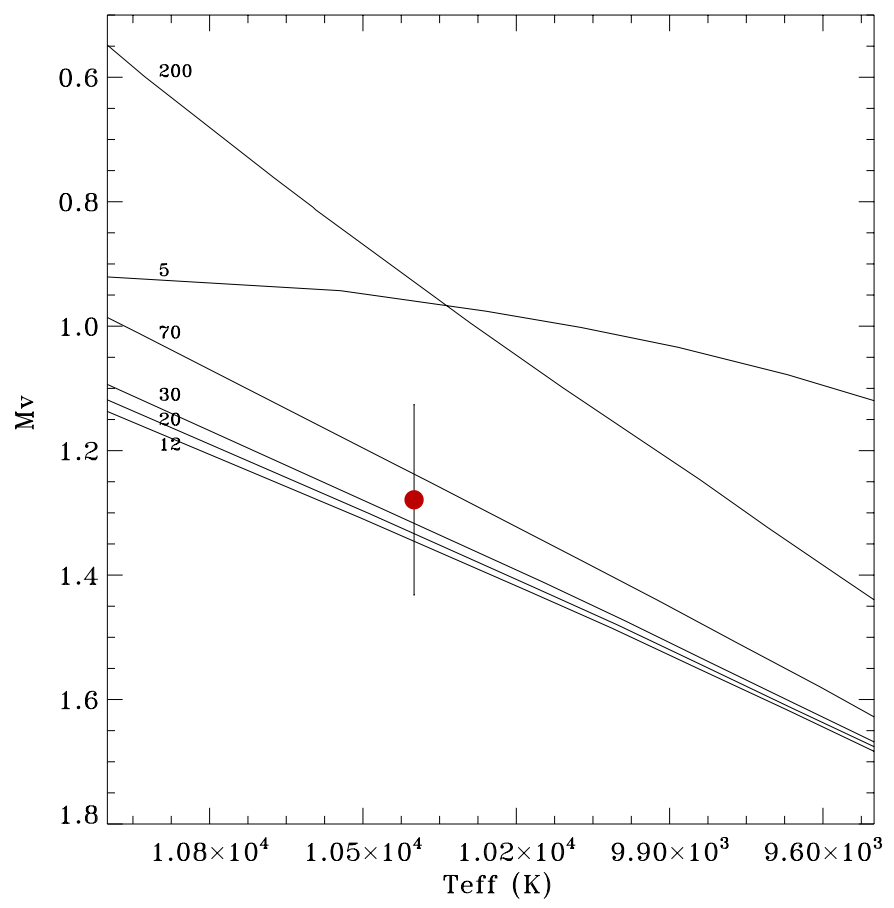

Fig. 1. The $V$-band absolute magnitude and effective temperature of HIP 64892 compared to predictions from the 5, 12, 20, 30, 70, and $200 \mathrm{Myr}$ isochrones of Bressan et al. (2012). The placement of HIP 64892 is consistent with the estimated local age of $16 \mathrm{Myr}$ within the measured uncertainties.

out an equal-luminosity binary down to a separation of about 40 mas.

By comparing the observed colours of HIP 64892 with those expected from the tables by Pecaut \& Mamajek (2013), we find they are consistent with the B9.5 spectral classification, and find a low reddening value of $\mathrm{E}(\mathrm{B}-\mathrm{V})=0.01$ consistent with the lack of $V$-band extinction found by Chen et al. (2012). In addition, the Chen et al. (2012) analysis showed no signs of an infrared excess. We explore the implications of this on the presence of dust in the system in Appendix A.

The Pecaut \& Mamajek (2013) tables predict an effective temperature of $10400 \mathrm{~K}$ for a B9.5 star. For such a hot star, the pre-main sequence isochrones collapse on the zero-age main sequence (ZAMS) in less than $10 \mathrm{Myr}$, and significant post-main sequence evolution is not expected for tens of Myr following this. When combined with the large uncertainty on the parallax of HIP 64892, this makes prediction of its age based on isochronal analysis difficult. The $\mathrm{V}$ magnitude and effective temperature of HIP 64892 are shown in Fig. 1, relative to the Bressan et al. (2012) isochrones at various ages between 5 and 200 Myr. Within the uncertainties, the placement of HIP 64892 is close to the ZAMS, and therefore consistent with LCC membership.

The Sco-Cen subgroups are known to show significant age spread. The recent age map by Pecaut \& Mamajek (2016) yields a value of $16 \mathrm{Myr}$ at the location of our target, equal to the commonly adopted age for the group. We adopt this value as the most likely age of HIP 64892, with the approximate ZAMS time as a lower limit. While an upper limit from comparison with the isochrones would be $\sim 100 \mathrm{Myr}$, we instead use the nearby star TYC 7780-1467-1 to place a more precise bound on the age. This star has a well-determined age of $20 \mathrm{Myr}$ calculated from comparison with isochrones and supported by its lithium abundance (EW Li $=360 \mathrm{m \AA}$; Torres et al. 2006) and fast rotation (rotation period 4.66 d; Kiraga 2012). Given this value, we think 
Table 2. Observing log.

\begin{tabular}{cccccccc}
\hline \hline UT Date & Instrument & Filter & $\begin{array}{c}\mathrm{DIT}^{a} \\
{[\mathrm{~s}]}\end{array}$ & $N_{\exp }^{a}$ & $\begin{array}{c}\Delta \pi^{a} \\
{\left[{ }^{\circ}\right]}\end{array}$ & $\begin{array}{c}\text { True North correction } \\
{\left[{ }^{\circ}\right]}\end{array}$ & $\begin{array}{c}\text { Plate scale } \\
{\left[\text { mas pixel }^{-1}\right]}\end{array}$ \\
\hline 2011-Jun-08 & NACO & $\mathrm{L}^{\prime}$ & 0.2 & 8000 & 130.4 & $-0.5 \pm 0.1$ & $27.1 \pm 0.05$ \\
2016-Apr-01 & IRDIS & H2/H3 & 64 & 64 & 45 & $-1.73 \pm 0.06$ & $12.255 \pm 0.009$ \\
2016-Apr-01 & IFS & YJ & 64 & 64 & 45 & $-102.21 \pm 0.06$ & $7.46 \pm 0.02$ \\
2017-Feb-08 & IRDIS & K1/K2 & 64 & 72 & 61 & $-1.71 \pm 0.06$ & $12.249 \pm 0.009$ \\
2017-Feb-08 & IFS & YH & 64 & 72 & 61 & $-102.19 \pm 0.06$ & $7.46 \pm 0.02$ \\
\hline
\end{tabular}

Notes. ${ }^{(a)}$ DIT refers to the integration time of each image, $N_{\text {exp }}$ to the total number of images obtained, and $\Delta \pi$ to the parallactic angle range during the sequence.

it unlikely that HIP 64892 is older than $~ 30$ Myr. We adopt an uncertainty of $15 \mathrm{Myr}$, leading to an age estimate of $16_{-7}^{+15}$. The stellar mass and radius from the Bressan et al. (2012) models are $2.35 \pm 0.09 M_{\odot}$ and $1.79 \pm 0.10 R_{\odot}$, respectively.

Due to its unknown rotational inclination and large measured $v \sin i$, HIP 64892 is expected to show significant oblateness. For this reason, any measurements of the stellar parameters may be influenced by its orientation. While a similar degeneracy between inclination and age caused issues for studies of other rapidly rotating stars such as $\kappa$ And (e.g. Hinkley et al. 2013; Jones et al. 2016), our age estimate relies on the firm Sco-Cen membership of HIP 64892, which is independent of these concerns. However, the effective temperature and mass in particular may be affected by the inclination of HIP 64892 .

\section{Observations and data reduction}

\subsection{SPHERE imaging}

The star HIP 64892 was observed with SPHERE on 2016-Apr-01 as part of the SHINE exoplanet survey. These data were taken with the IRDIFS mode, and are summarised in Table 2. After a bright companion candidate was discovered in these data, follow-up observations were taken on 2017-Feb-08 using the IRDIFS_EXT mode to confirm its co-moving status and extend the spectral coverage.

These modes allow the Integral Field Spectrograph (IFS; Claudi et al. 2008) and Infra-Red Dual-band Imager and Spectrograph (IRDIS; Dohlen et al. 2008) modules to be used simultaneously through the use of a dichroic. In these configurations, IFS provides a low-resolution spectrum $(R \sim 55$ across $Y-J$ bands or $R \sim 35$ across $Y-H$ bands) while IRDIS operates in dual-band imaging mode (Vigan et al. 2010).

For each observing sequence, several calibration frames were taken at the beginning and end of the sequence. These consisted of unsaturated short exposure images to estimate the flux of the primary star and as a point spread function (PSF) reference, followed by a sequence of images with a sinusoidal modulation introduced to the deformable mirror to generate satellite spots used to calculate the position of the star behind the coronagraph. The majority of the observing sequence consisted of long exposure (64 s) coronagraphic imaging.

For the 2017 data, the satellite spots were used for the entirety of the coronagraphic imaging sequence rather than a separate set of frames at the beginning and end. This allowed us to correct for changes in flux and the star's position during the observations, at the cost of a small contrast loss at the separation of the satellite spots.

The data were reduced using the SPHERE data reduction and handling (DRH) pipeline (Pavlov et al. 2008) to perform the basic image cleaning steps (background subtraction, flat fielding, removal of bad pixels, calculation of the star's position behind the coronagraph, as well as extraction of the spectral data cubes for IFS). To process the IFS data, the DRH routines were augmented with additional routines from the SPHERE Data Centre to reduce the spectral cross-talk and improve the wavelength calibration and bad pixel correction (Mesa et al. 2015). Both IFS and IRDIS data were corrected using the astrometric calibration procedures described in Maire et al. (2016b).

A bright companion candidate is clearly visible in the raw coronagraphic frames, at a separation of $1.270 \pm 0.002^{\prime \prime}$ in the 2016 dataset $(159 \pm 12 \mathrm{AU}$ in projected physical separation). This separation places it outside of the field of view of the IFS module, and so we report the results from IRDIS only. To extract the astrometry and photometry of this object, a classical angular differential imaging procedure (ADI; Marois et al. 2006) was applied to remove the contribution from the primary star while minimising self-subtraction and other systematic effects that may be introduced by more aggressive PSF subtraction techniques. The ADI procedure and calculation of the relative astrometry and photometry of the companion were accomplished using the Specal pipeline developed for the SHINE survey (R. Galicher, 2018, in prep.). The final reduced images from this approach are shown in Fig. 2.

The astrometry was measured using the negative companion injection technique (Lagrange et al. 2010), where the mean unsaturated PSF of the primary star was subtracted from the raw frames. The position and flux of the injected PSF was varied to minimise the standard deviation inside a 3 FWHM (full width at half maximum) diameter region around the companion in the final ADI processed image. Once the best-fit values were found, each parameter was varied until the standard deviation increased by a factor of 1.15 . This value was empirically calculated to correspond to $1 \sigma$ uncertainties across a range of potential companion and observational parameters. A systematic uncertainty of 2 mas on the star position was adopted for the 2016 dataset, which dominates the astrometric uncertainty budget. The resulting astrometric and photometric measurements are given in Table 3.

A second reduction of each dataset was performed with the aim of searching for companions at higher contrasts. For the IRDIS datasets, the TLOCI algorithm was used (Marois et al. 2014), while for the IFS datasets we used the PCA-based angular and spectral differential imaging algorithm described in Mesa et al. (2015). The resulting detection limits are shown in Fig. 3. Apart from the bright companion at 1.27", we find no evidence of companions at smaller separations. Three additional objects were detected at much larger separations, and are discussed in Sect. 4.1. 


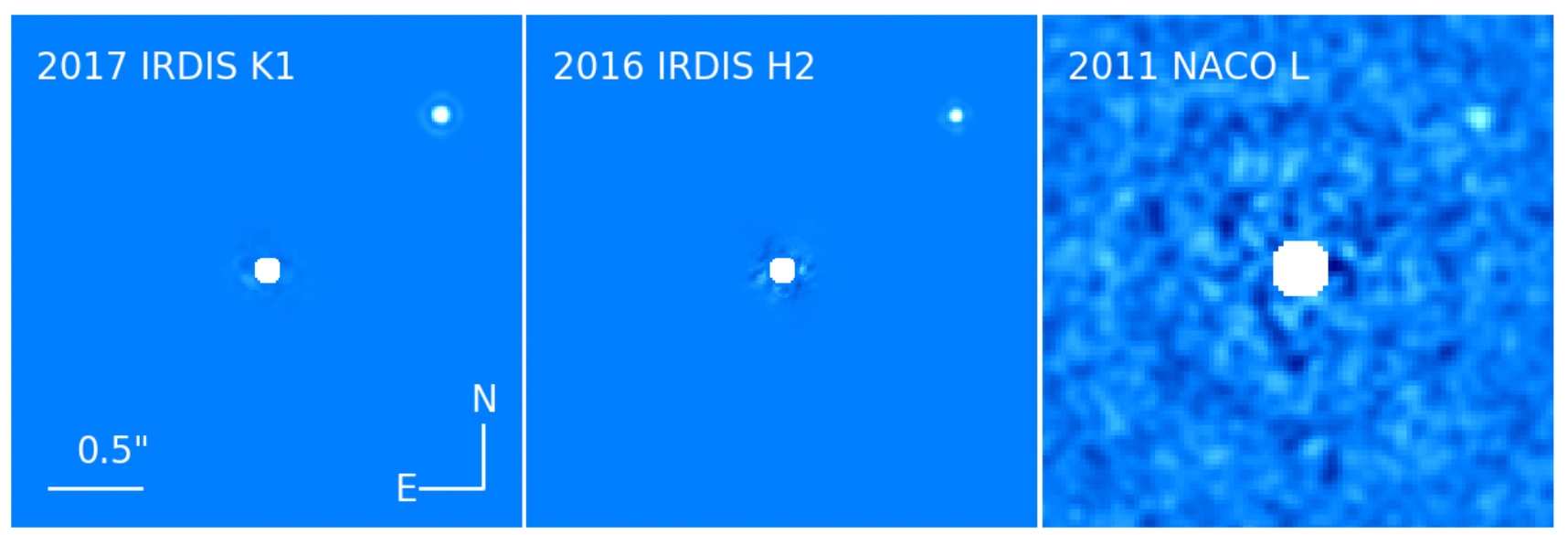

Fig. 2. ADI-processed images from the two SPHERE-IRDIS datasets taken with the K1 and $\mathrm{H} 2$ filters, and the NACO data taken with the $\mathrm{L}^{\prime}$ filter. The companion is detected with $S / N>1000$ in the two SPHERE epochs, and $S / N>8$ in the NACO data.

Table 3. Observed astrometry and photometry of HIP 64892 B.

\begin{tabular}{cccccccc}
\hline \hline UT date & Instrument & Filter & $\rho\left({ }^{\prime \prime}\right)$ & $\theta\left(^{\circ}\right)$ & Contrast $(\mathrm{mag})$ & Abs. mag & Mass (COND) \\
\hline 2011-Jun-08 & NACO & $\mathrm{L}^{\prime}$ & $1.272 \pm 0.029$ & $310.0 \pm 1.3$ & $6.10 \pm 0.08$ & $7.61 \pm 0.17$ & $37 \pm 9$ \\
2016-Apr-01 & IRDIS & H2 & $1.2703 \pm 0.0023$ & $311.68 \pm 0.15$ & $7.23 \pm 0.08$ & $8.73 \pm 0.17$ & $29 \pm 4$ \\
2016-Apr-01 & IRDIS & H3 & $1.2704 \pm 0.0022$ & $311.69 \pm 0.15$ & $6.99 \pm 0.08$ & $8.46 \pm 0.17$ & $29 \pm 5$ \\
2017-Feb-08 & IRDIS & K1 & $1.2753 \pm 0.0010$ & $311.74 \pm 0.12$ & $6.80 \pm 0.08$ & $8.29 \pm 0.17$ & $34 \pm 7$ \\
2017-Feb-08 & IRDIS & K2 & $1.2734 \pm 0.0010$ & $311.77 \pm 0.12$ & $6.49 \pm 0.12$ & $7.97 \pm 0.19$ & $35 \pm 8$ \\
\hline
\end{tabular}

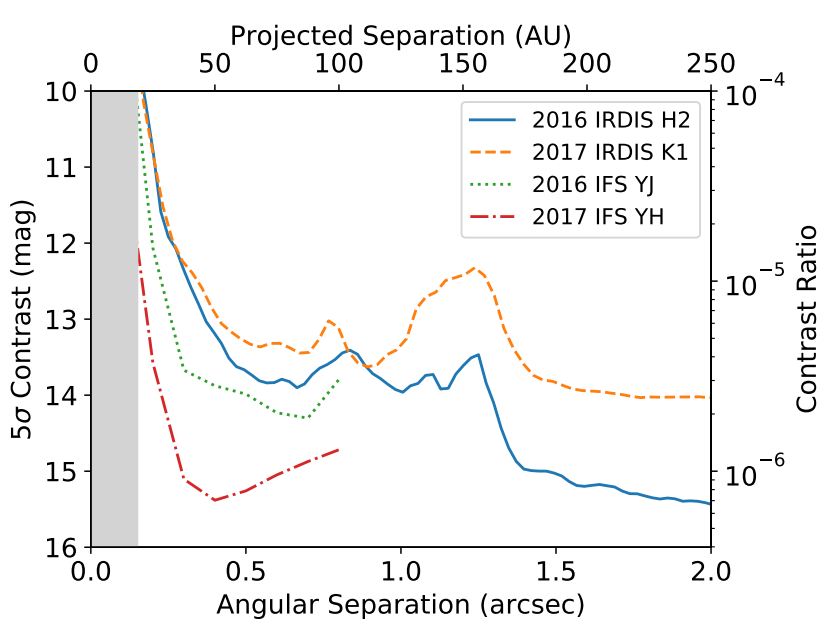

Fig. 3. Detection limits for the two SPHERE datasets, after processing using the TLOCI algorithm. The grey shaded region indicates the separations partially or fully blocked by the coronagraph.

\subsection{SPHERE long slit spectroscopy}

The star HIP 64892 was also observed with SPHERE IRDIS long slit spectroscopy (LSS; Vigan et al. 2008) on 2017-Mar-18. These observations utilised the medium resolution spectroscopy mode, which covers wavelengths from 0.95 to $1.65 \mu \mathrm{m}$ with a spectral resolution of $R \sim 350$. The observing sequence consisted of a series of alternate images with the companion inside and outside of the slit. To move the companion outside of the slit, a small offset is applied on the derotator so as to keep the star centred on the coronagraph. This strategy has been demonstrated to be very efficient to build and subtract reference images of the speckles and stellar halo while minimising the self-subtraction effects on the spectrum of the companion (Vigan et al. 2016).
Additional sky backgrounds were also obtained at the end of the sequence along with an unsaturated spectrum of the star to serve as reference for the contrast.

The data were reduced using the SILSS pipeline (Vigan 2016). This pipeline combines recipes from the standard ESO pipeline with custom $I D L$ routines. Briefly, data are background subtracted, flat fielded, and corrected for bad pixels. The wavelength calibration is performed and the data are corrected for the slight tilt of the grism, which causes a change in the position of the PSF with wavelength. Finally, the speckles are subtracted using principal component analysis, with the modes constructed from the spectra obtained with the companion outside of the slit.

\subsection{Archival NACO sparse aperture masking data}

In addition to the SPHERE data, we utilised archival sparse aperture masking (SAM) data from the VLT-NACO instrument, taken on 2011-Jun-08 (Program 087.C-0790(A), PI: Ireland). While the main purpose of the SAM mode is to detect companions and resolve structures at small angular separations (typically $<300$ mas), this does not preclude the detection of bright objects at larger separations. The data were taken with the L' filter in pupil tracking mode, and were split into blocks of 1600 exposures of $0.2 \mathrm{~s}$ each. Each of the five blocks was interspersed amongst observations of other targets.

Rather than process the data in an interferometric framework, we treated it as a traditional ADI dataset to focus on larger separations. The data were processed using the GRAPHIC pipeline (Hagelberg et al. 2016). The data were sky subtracted, flat fielded, cleaned of bad pixels, centred on the primary star, and stacked by binning 200 frames at a time. A Gaussian fit was performed to each PSF to calculate the position of the star, since it provides a reasonable match to the core of the SAM PSF. A python implementation of the KLIP algorithm (Soummer et al. 2012) was then applied, where the first 15 modes were removed, 

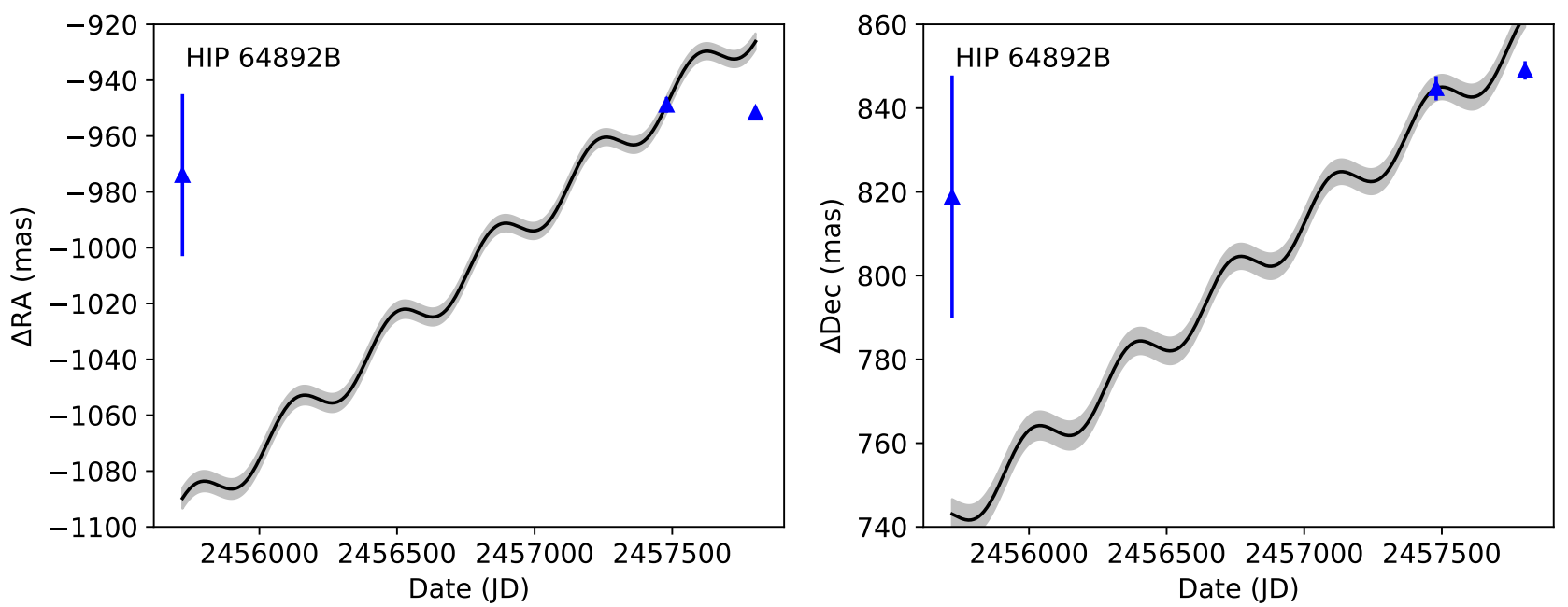

Fig. 4. Measured position of HIP 64892B relative to HIP 64892A. The 2011 NACO L', 2016 IRDIS H2, and 2017 IRDIS K1 positions are shown with blue triangles. The predicted motion for a stationary background object relative to the 2016 position is marked by the black line, with its uncertainty represented by the grey shaded region. The observed positions of HIP 64892B strongly conflict with the predictions for a background object, suggesting that it is co-moving.

Table 4. Additional objects detected by SPHERE-IRDIS.

\begin{tabular}{cccccccc}
\hline \hline Candidate & UT Date & Filter & $\rho(\mathrm{mas})$ & $\theta(\mathrm{deg})$ & $\Delta$ RA $(\mathrm{mas})$ & $\Delta$ Dec. $(\mathrm{mas})$ & Contrast (mag) \\
\hline 1 & 2016-Apr-01 & H2 & $6000 \pm 3$ & $201.86 \pm 0.12$ & $-2234 \pm 12$ & $-5568 \pm 5$ & $10.5 \pm 0.1$ \\
1 & 2017-Feb-08 & K1 & $5978 \pm 4$ & $201.60 \pm 0.07$ & $-2201 \pm 7$ & $-5558 \pm 5$ & $10.7 \pm 0.1$ \\
2 & 2016-Apr-01 & H2 & $5865 \pm 5$ & $202.86 \pm 0.12$ & $-2278 \pm 12$ & $-5404 \pm 7$ & $13.7 \pm 0.1$ \\
3 & 2016-Apr-01 & H2 & $7003 \pm 6$ & $186.89 \pm 0.12$ & $-840 \pm 14$ & $-6952 \pm 6$ & $10.2 \pm 0.1$ \\
\hline
\end{tabular}

resulting in the redetection of the SPHERE companion. The final reduced image is shown in the right panel of Fig. 2.

To calculate the position and flux of the companion, we used the negative companion injection technique applied to the data cube after binning. We chose to minimise the square of the residuals within a circle of radius $\lambda / D$ centred on the companion peak calculated from the first reduced image. To explore the likelihood function, we used emcee (Foreman-Mackey et al. 2013), a python implementation of the affine-invariant Markov chain Monte Carlo ensemble sampler. Due to the lack of detailed studies on potential systematic biases introduced into the companion astrometry by applying this technique to SAM data, we conservatively added an additional uncertainty to the companion position of one pixel (27.1 mas). Of particular concern is the way in which the time-varying fine structure of the large SAM PSF may influence the calculation of the star's position.

\section{Results}

\subsection{Astrometry}

To confirm the co-moving status of HIP 64892B, we compared its position relative to HIP $64892 \mathrm{~A}$ between the datasets. We used the astrometry from the 2016 SPHERE dataset with the parallax and proper motion of HIP 64892 to predict the position expected for a background object at the 2011 and 2017 epochs. The result is shown in Fig. 4.

For HIP 64892B, the observed positions in 2011 and 2017 differ from the prediction for a background object by $4 \sigma$ and $8 \sigma$ respectively. Instead, we find a lack of significant relative motion. This clearly shows that the object is co-moving with HIP 64892A.
The angular separation of HIP 64892B corresponds to a projected separation of $159 \pm 12 \mathrm{AU}$. With the primary star mass of $2.35 M_{\odot}$ we would expect an orbital period of the order of $\sim 10^{3} \mathrm{yr}$. This is consistent with the lack of significant motion in the measured astrometry, and suggests that an additional epoch in 2018 or later may show clear orbital motion and help to constrain the orbital parameters of the companion.

In addition to HIP 64892B, three objects were detected at larger separations in the IRDIS field of view. Their astrometry and photometry are given in Table 4 . Candidate 1 was detected at high significance in both epochs, while the other two were not detected in the 2017-Feb-08 $K$-band data. The relative motion of candidate 1 between the two epochs is shown in Fig. 5 and is similar to that expected from a background star. Its position differs from the prediction by $1.3 \sigma$, while it is inconsistent with a co-moving object at $2.8 \sigma$, showing that it is likely to be a background star.

The IRDIS $\mathrm{H} 2$ and $\mathrm{H} 3$ photometry of the remaining two objects indicates that they are also likely to be background stars. If located at the same distance as HIP 64892, their H2 magnitudes and $\mathrm{H} 2-\mathrm{H} 3$ colours would be inconsistent with those of other known objects. Their $\mathrm{H} 2$ magnitudes would be consistent with those of $\mathrm{T}$ dwarfs, but without significant methane absorption that would be measurable in their $\mathrm{H} 2-\mathrm{H} 3$ colours.

\subsection{Mass}

To estimate the mass of HIP 64892B, we first converted each of the apparent photometric fluxes for the companion (IRDIS $\mathrm{H} 2, \mathrm{H} 3, \mathrm{~K} 1, \mathrm{~K} 2$, and NACO L') into absolute magnitudes using the distance of $125 \pm 9 \mathrm{pc}$. To calculate the photometric zeropoint of each filter, we used the spectrum of Vega from 

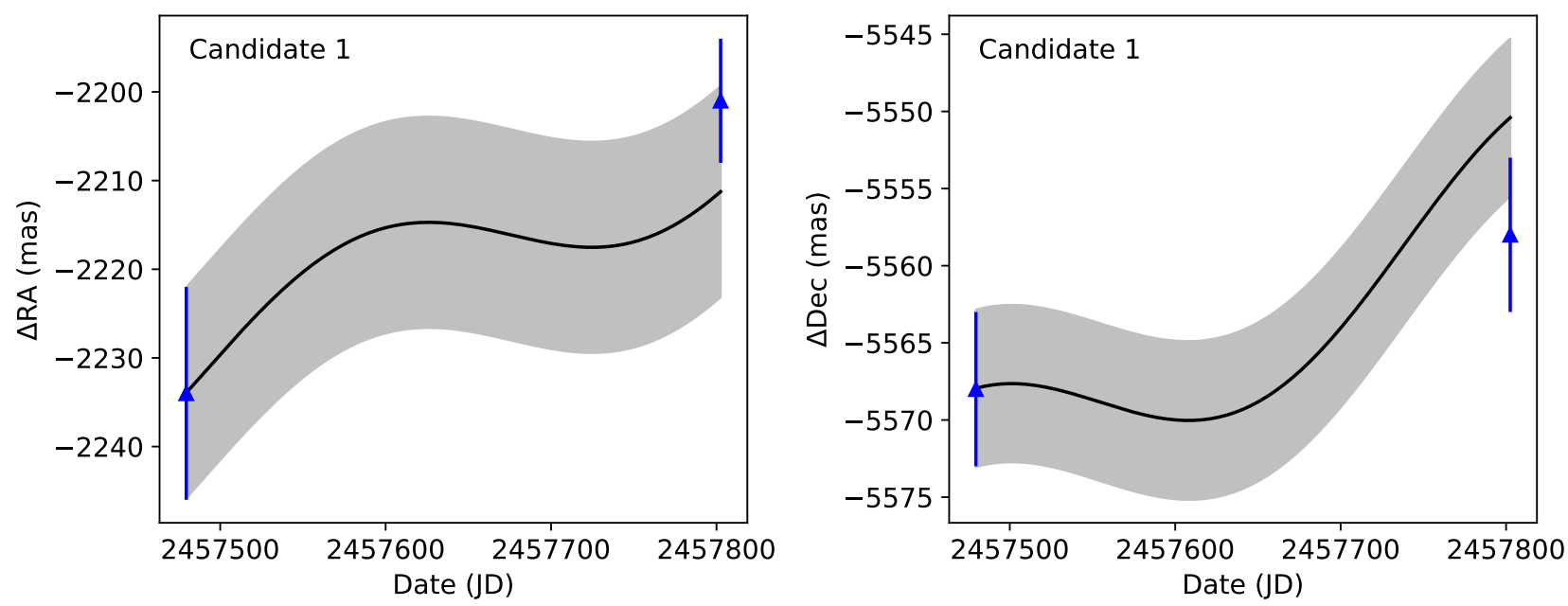

Fig. 5. Same as Fig. 4, showing the measured position of companion candidate 1 with respect to HIP 64892A. This object agrees with the prediction for a background object.

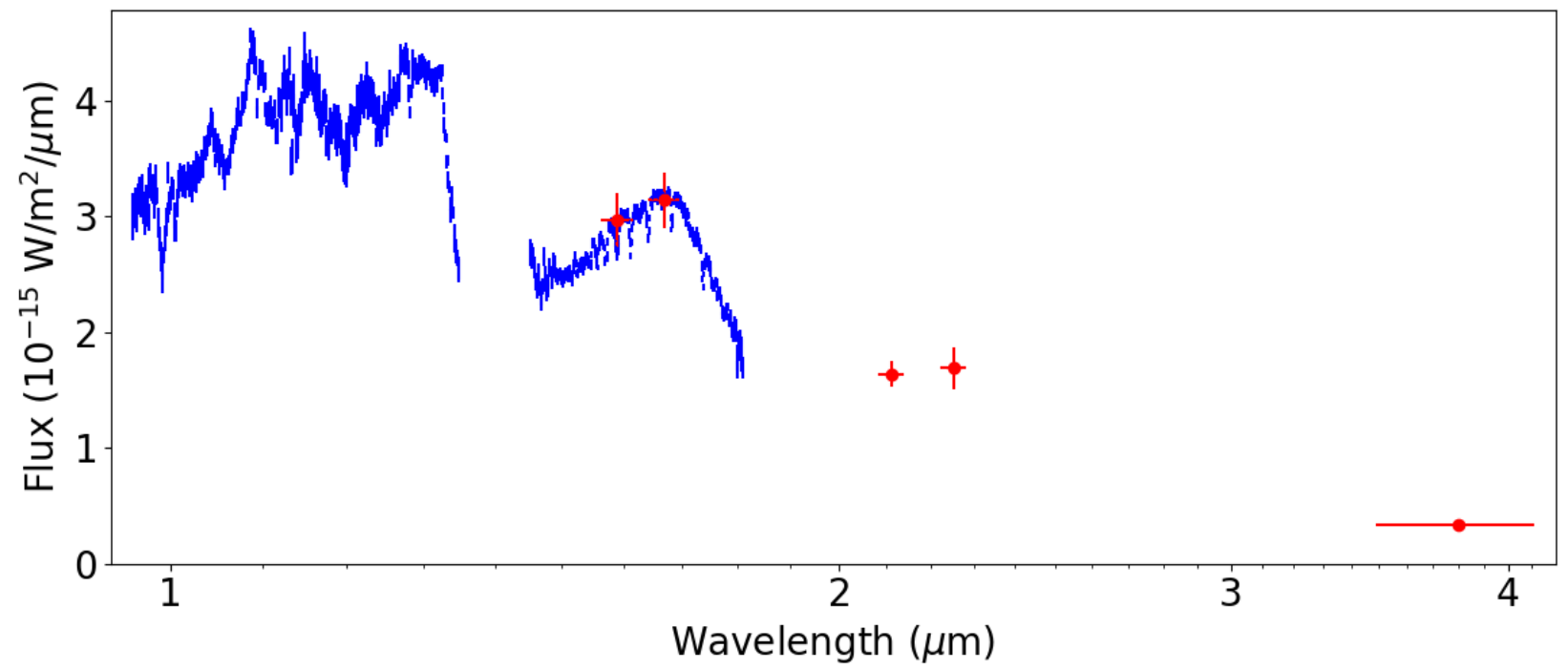

Fig. 6. Observed spectrum of HIP 64892B. SPHERE IRDIS LSS data are shown in blue, while the SPHERE IRDIS and NACO photometric measurements are in red. The errorbars on the $x$-axis of the photometric measurements represent the FWHM of the filter used.

Bohlin (2007) along with the filter transmission profiles from each instrument. We then interpolated the COND evolutionary models (Baraffe et al. 2003) using the age and absolute magnitudes to yield estimates of the mass of HIP 64892B. The measurements, listed in Table 3, are consistent with masses of 29-37 $M_{\mathrm{J}}$. This implies a mass ratio between the brown dwarf and the primary of $q \sim 0.014$.

\subsection{Spectral properties}

We produced a spectral model for the primary by scaling a BT-Settl spectrum with $T_{\text {eff }}=10400 \mathrm{~K}, \log g=4,[M / H]=$ -0.5 using photometric measurements compiled from 2MASS, Tycho-2, and WISE (Høg et al. 2000; Skrutskie et al. 2006; Wright et al. 2010). This was then used to convert the contrast measurements from IRDIS and NACO to apparent fluxes. The resulting spectrum is shown in Fig. 6.

To estimate the spectral type and effective temperature of the companion, we compared the observed spectrum and photometry of HIP 64892B with a range of spectra compiled from the literature, using the goodness-of-fit statistic $G$ (e.g. Cushing et al. 2008). We considered young $\mathrm{L}$ dwarfs from the Upper-Scorpius subgroup (Lodieu et al. 2008) as well as companions of Upper Scorpius stars (Lafrenière et al. 2008; Lachapelle et al. 2015). We also compared the companion spectrum to those of young free floating objects from the Montreal ${ }^{3}$ (Robert et al. 2016; Gagné et al. 2014a,b, 2015a) and Allers \& Liu (2013) libraries, and to libraries of medium-resolution spectra of old M, L, and T field dwarfs (Burgasser et al. 2002; McLean et al. 2003; Cushing et al. 2005; Rayner et al. 2009). The result is plotted in Fig. 7 for a range of objects covering different ages, masses, and spectral types. We find the best matches are given by young, low surface gravity objects with spectral types close to M9. From this, we adopt a spectral type of M9 \pm 1 for HIP 64892B.

In Fig. 8, we show the comparison of the observed spectrum of HIP 64892B to those of young and old field dwarfs at the M/L transition. Several features in the spectrum are indicative of a young object. The doublets of gravity-sensitive potassium bands at $1.169 / 1.177 \mu \mathrm{m}$ and $1.243 / 1.253 \mu \mathrm{m}$ are reduced. The $H$-band has a triangular shape characteristic of low gravity atmospheres. Visually, the spectrum agrees well with those of $\mathrm{M} 9_{\gamma}$ candidate

\footnotetext{
3 https://jgagneastro.wordpress.com/the-montrealspectral-library/
} 
A. Cheetham et al.: Discovery of a brown dwarf companion to the star HIP 64892

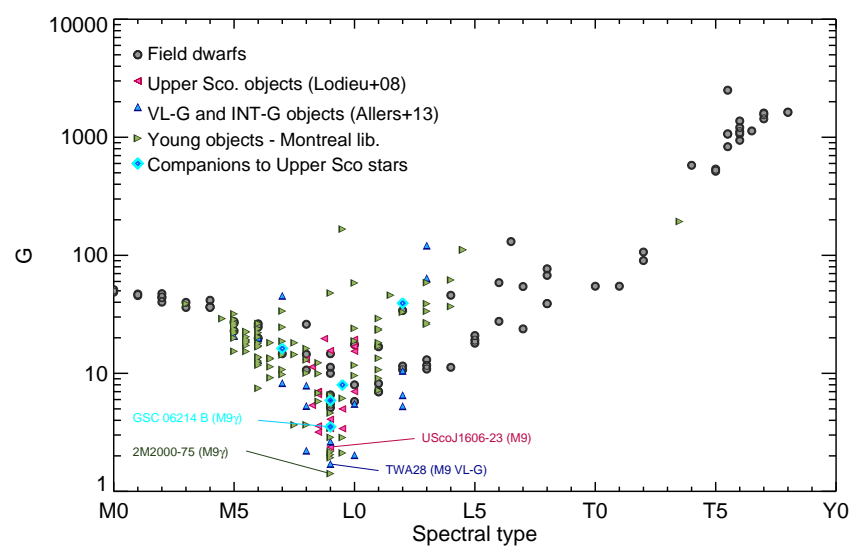

Fig. 7. Goodness-of-fit statistic $G$ plotted as a function of spectral type for a number of objects in the range M0-T8. A clear $G$ minimum is seen at late $\mathrm{M}$ spectral types and the best matches are given by young, low surface gravity objects with spectral types of M9.

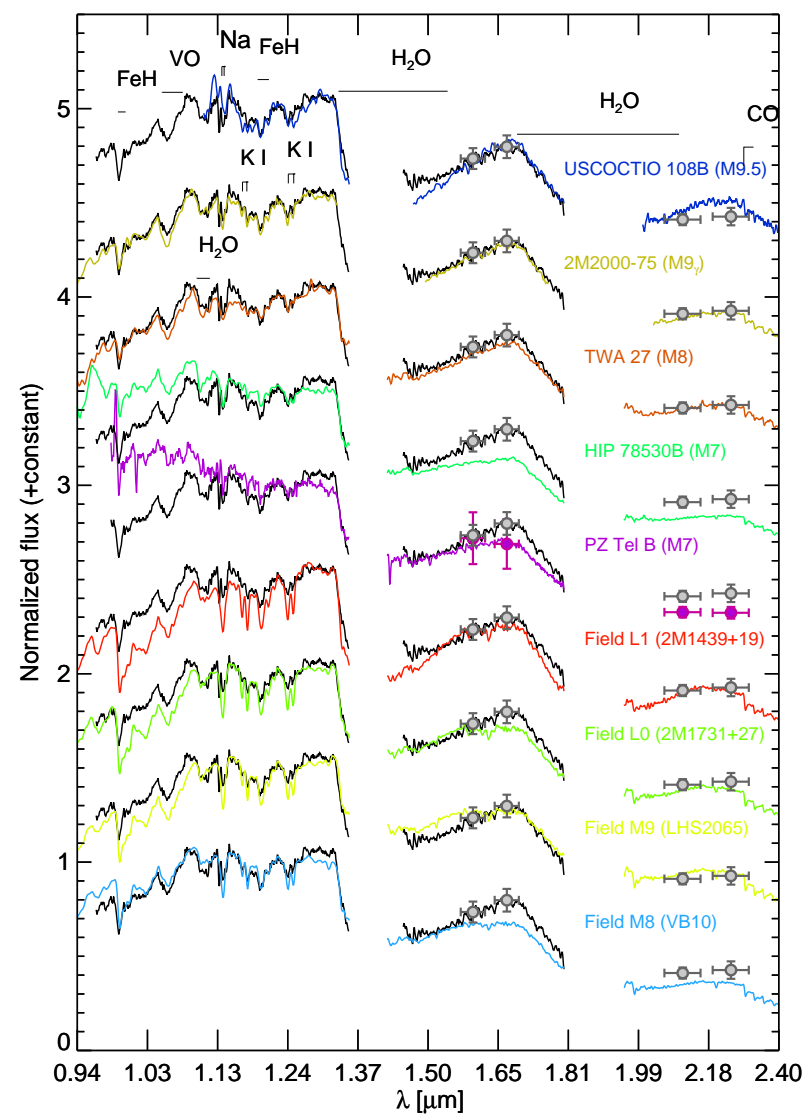

Fig. 8. Comparison of the observed spectrum (in black) with field dwarfs and similar young low-mass brown dwarf companions (coloured lines). The measured photometric points from the IRDIS filters are shown with grey circles. The M9 object 2MASS J20004841-7523070 (2M2000-75) provides the best match to the measured spectrum of HIP $64892 B$

members of the $\beta$ Pictoris and Argus moving groups (2050 Myr; Gagné et al. 2015b), with 2MASS J20004841?7523070 giving the best fit.

As seen in Fig. 8, the spectrum of HIP 64892B is also well reproduced by the spectrum of the 8 Myr-old M8 dwarf 2M1207A (TWA27) from the TW Hydrae association, which shows many of the same features seen in our data. When

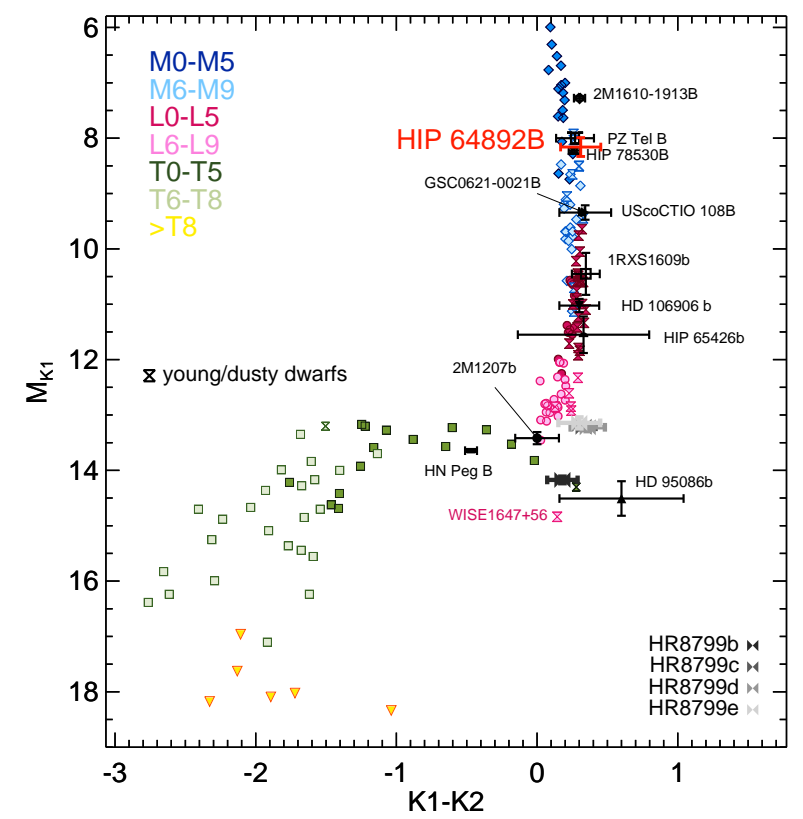

Fig. 9. Colour-magnitude diagram comparing K1 absolute magnitudes and $\mathrm{K} 1-\mathrm{K} 2$ colours of a range of low-mass stellar and substellar objects from the literature. Synthetic K1 and K2 fluxes were computed for each target from published spectra. A number of young and dusty companions observed with SPHERE or with published $K$-band spectra were added to the diagram. HIP 64892B falls in a similar location to PZ Tel B and HIP 78530B, both young brown dwarfs with late-M spectral types.

compared to the SPHERE-LSS data of the M7 companion PZ Tel B (Maire et al. 2016a), we can see a clear difference in slope indicating a later spectral type for HIP 64892B. Also shown are the objects UScoCTIO 108B (Béjar et al. 2008), HIP 78530B (Lafrenière et al. 2011), and several field dwarfs. Using the empirical relation between spectral type and effective temperature for young objects with low surface gravity from Filippazzo et al. (2015), our spectral type constraints correspond to an effective temperature of $T_{\text {eff }}=2600 \pm 300 \mathrm{~K}$.

From the flux-calibrated LSS spectrum, we derived a synthetic absolute magnitude of $M_{\mathrm{J}, 2 \mathrm{MASS}}=9.37 \pm 0.15 \mathrm{mag}$. Combining this with the spectral type of M9 \pm 1 , we can use the bolometric correction relations from Filippazzo et al. (2015) to derive a bolometric luminosity of $\log \left(L / L_{\odot}\right)=-2.66 \pm 0.10$ dex. In addition, we converted the $\mathrm{K} 1$ flux measurement to a predicted $K_{\mathrm{S}, 2 \mathrm{MASS}}$ absolute magnitude using the SpeX spectrum of TWA 27A as an analog. The resulting value of $M_{\mathrm{Ks}, 2 \mathrm{MASS}}=$ $8.02 \pm 0.17 \mathrm{mag}$ corresponds to a luminosity of $\log \left(L / L_{\odot}\right)=$ $-2.51 \pm 0.11$ dex using the same method.

We also compared the observed spectrum to the BT-Settl model grid (Baraffe et al. 2015) as a function of $T_{\text {eff }}, \log g$, and radius $R$. We find a best-fit temperature and radius of $T_{\text {eff }}=$ $2600 \pm 100 \mathrm{~K}$ and $R=2.3 \pm 0.14 R_{\mathrm{J}}$, similar to those predicted by the COND models for a $33 M_{\mathrm{J}}$ object with an age of $16 \mathrm{Myr}$. We find that the $\log g$ value is poorly constrained, with a best-fit value $\log g=5.5$. When combined with the radius, this predicts a much larger mass than expected. However, gravity-sensitive features are generally narrow and fitting to the entire spectrum at once may complicate this measurement. To investigate this, we performed the same fit to the two sections of the LSS spectrum either side of the $1.4 \mu \mathrm{m}$ telluric feature individually. The $Y-J$ band spectrum gives an estimate of $\log g=4.0 \pm 0.5$, while the $H$-band spectrum yields $\log g=3.5 \pm 1.0$, indicating that a lower value is likely. 
The spectral type estimate and low surface gravity are also supported by the position of HIP 64892B on colour-magnitude diagrams. In Fig. 9 we show its K1 magnitude and K1-K2 colour compared to a range of field objects assembled from the SpeX Prism Library (Burgasser 2014) and from Leggett et al. (2000). For these objects we generated synthetic photometry using the SPHERE filter bandpasses. In addition, we also show a range of young companions with SPHERE K1K2 photometry or $K$-band spectra from the literature (Luhman et al. 2007; Lafrenière et al. 2008; Patience et al. 2010; Bonnefoy et al. 2014a; Lachapelle et al. 2015; Lagrange et al. 2016; Maire et al. 2016a; Zurlo et al. 2016; Chauvin et al. 2017, 2018).

The position of HIP 64892B is similar to PZ Tel B and HIP $78530 \mathrm{~B}$, both young companions with late-M spectral types. All three lie close to the mid-M sequence of field dwarfs, showing a slight over-luminosity compared to late-M field objects that match their spectral types. This trend is also seen in young field brown dwarfs (Faherty et al. 2012; Liu et al. 2013).

\section{Discussion and conclusion}

The star HIP 64892 joins a growing number of high or intermediate mass stars with extreme mass ratio companions at large separations $(<10 \%,>10 \mathrm{AU})$. While brown dwarf companions to solar-type stars from both RV and imaging surveys are inherently rare (the so-called "brown dwarf desert"; Grether \& Lineweaver 2006; Kraus et al. 2008; Metchev \& Hillenbrand 2009), evidence suggests that the occurrence rates of companions around intermediate mass stars may be substantially higher (Vigan et al. 2012). This trend is seen for companions with masses spanning the planetary to stellar regimes (e.g. Johnson et al. 2010; Janson et al. 2013).

Despite this, HIP 64892 is one of the highest mass stars around which a substellar companion has been detected, due to the challenges associated with observing such stars and the tendency for large surveys to focus on solar-like or low-mass stars. Only HIP 78530B (Lafrenière et al. 2011), $\kappa$ And b (Carson et al. 2013), and HIP 77900B (Aller et al. 2013) orbit stars with a larger mass.

The large mass of the primary leads to a mass ratio for HIP $64892 \mathrm{~B}$ that is particularly small $(q \sim 0.014)$. For low-mass and solar-like stars, such a value would correspond to objects at or below the deuterium burning limit, making HIP 64892B a valuable object for studying the overlapping processes of binary star and planet formation in the brown dwarf regime. Indeed, the properties of HIP 64892B raise the possibility that it formed via gravitational instability, either through a binary-star mechanism or through disk instability in the circumstellar disk of the primary (Boss 1997). We investigate the latter idea further in Appendix B, finding that the observed mass and separation of HIP 64892B are compatible with in situ formation via disk instability. This process is one of the proposed pathways for giant planet formation, making HIP 64892B an important object for understanding this mechanism.

The brown dwarf HIP 64892B also stands to be an important object for calibrating substellar formation and evolutionary models, with a well-known age tied to that of the LCC association, and its brightness allowing high signal-to-noise ratio $(\mathrm{S} / \mathrm{N})$ spectroscopic and photometric measurements. While the spectroscopic and photometric measurements presented here have high $\mathrm{S} / \mathrm{N}$, the uncertainty on the distance is relatively large, and dominates the uncertainties on the absolute magnitudes, luminosity, and isochronal mass for HIP 64892B. A much more precise distance measurement will come from the Gaia parallax, which should be included in the second data release.

Many of the derived properties of the newly discovered companion match those of the planet-hosting brown dwarf TWA 27, with an excellent match found between their spectra. This object may prove to be a useful analogue and a point of direct comparison between young brown dwarfs in single and multiple systems.

The HIP 64892 system as a whole has many parallels to that of $\kappa$ And. While the stellar hosts have a similar mass and spectral type, the companion HIP 64892B appears to be a hotter, younger, and higher-mass analogue of $\kappa$ And B (Hinkley et al. 2013; Bonnefoy et al. 2014b; Jones et al. 2016). When combined with HIP 78530B (Lafrenière et al. 2011), HR 3549B (Mawet et al. 2015), HD 1160 (Nielsen et al. 2012), and $\eta$ Tel B (Lowrance et al. 2000), they form a useful sample to explore the properties of low and intermediate-mass brown dwarf companions to young, 2-3 $M_{\odot}$ stars.

Acknowledgements. This work has been carried out within the frame of the National Centre for Competence in Research "PlanetS" supported by the Swiss National Science Foundation (SNSF). SPHERE is an instrument designed and built by a consortium consisting of IPAG (Grenoble, France), MPIA (Heidelberg, Germany), LAM (Marseille, France), LESIA (Paris, France), Laboratoire Lagrange (Nice, France), INAF - Osservatorio di Padova (Italy), Observatoire Astronomique de l'Université de Genève (Switzerland), ETH Zurich (Switzerland), NOVA (The Netherlands), ONERA (France), and ASTRON (The Netherlands) in collaboration with ESO. SPHERE was funded by ESO, with additional contributions from CNRS (France), MPIA (Germany), INAF (Italy), FINES (Switzerland), and NOVA (The Netherlands). SPHERE also received funding from the European Commission Sixth and Seventh Framework Programmes as part of the Optical Infrared Coordination Network for Astronomy (OPTICON) under grant number RII3-Ct-2004-001566 for FP6 (2004-2008), grant number 226604 for FP7 (2009-2012), and grant number 312430 for FP7 (2013-2016). This work has made use of the SPHERE Data Centre, jointly operated by OSUG/IPAG (Grenoble), PYTHEAS/LAM/CeSAM (Marseille), OCA/Lagrange (Nice), and Observatoire de Paris/LESIA (Paris) and supported by a grant from Labex OSUG@2020 (Investissements d'avenir - ANR10 LABX56). This publication makes use of data products from the Two Micron All Sky Survey, which is a joint project of the University of Massachusetts and the Infrared Processing and Analysis Center/California Institute of Technology, funded by the National Aeronautics and Space Administration and the National Science Foundation. This publication makes use of VOSA, developed under the Spanish Virtual Observatory project supported from the Spanish MICINN through grant AyA2011-24052. R.G., R.C., S.D. acknowledge support from the "Progetti Premiali" funding scheme of the Italian Ministry of Education, University, and Research. J.O. acknowledges financial support from ICM Núcleo Milenio de Formación Planetaria, NPF. Q.K. acknowledges funding from STFC via the Institute of Astronomy, Cambridge Consolidated Grant.

\section{References}

Aller, K. M., Kraus, A. L., Liu, M. C., et al. 2013, ApJ, 773, 63

Allers, K. N., \& Liu, M. C. 2013, ApJ, 772, 79

Baehr, H., Klahr, H., \& Kratter, K. M. 2017, ApJ, 848, 40

Baraffe, I., Chabrier, G., Barman, T. S., Allard, F., \& Hauschildt, P. H. 2003, A\&A, 402, 701

Baraffe, I., Homeier, D., Allard, F., \& Chabrier, G. 2015, A\&A, 577, A42

Bayo, A., Rodrigo, C., Barrado Y Navascués, D., et al. 2008, A\&A, 492, 277

Béjar, V. J. S., Zapatero Osorio, M. R., Pérez-Garrido, A., et al. 2008, ApJ, 673, L185

Beuzit, J.-L., Feldt, M., Dohlen, K., et al. 2008, Proc. SPIE, 7014, 701418

Bohlin, R. C. 2007, ASP Conf. Ser., 364, 315

Bonnefoy, M., Chauvin, G., Lagrange, A.-M., et al. 2014a, A\&A, 562, A127

Bonnefoy, M., Currie, T., Marleau, G.-D., et al. 2014b, A\&A, 562, A111

Boss, A. P. 1997, Science, 276, 1836

Bowler, B. P., Johnson, J. A., Marcy, G. W., et al. 2010, ApJ, 709, 396

Bowler, B. P., Liu, M. C., Shkolnik, E. L., \& Tamura, M. 2015, ApJS, 216, 7

Brahm, R., Jordán, A., \& Espinoza, N. 2017, PASP, 129, 034002

Bressan, A., Marigo, P., Girardi, L., et al. 2012, MNRAS, 427, 127

Burgasser, A. J. 2014, Astron. Soc. India Conf. Ser., 11, 7

Burgasser, A. J., Kirkpatrick, J. D., Brown, M. E., et al. 2002, ApJ, 564, 421 
Burns, J. A., Lamy, P. L., \& Soter, S. 1979, Icarus, 40, 1

Carson, J., Thalmann, C., Janson, M., et al. 2013, ApJ, 763, L32

Chauvin, G., Desidera, S., Lagrange, A.-M., et al. 2017, A\&A, 605, L9

Chauvin, G., Gratton, R., Bonnefoy, M., et al. 2018, A\&A, in press, DOI: 10.1051/0004-6361/201732077

Chen, C. H., Pecaut, M., Mamajek, E. E., Su, K. Y. L., \& Bitner, M. 2012, ApJ, 756, 133

Chini, R., Hoffmeister, V. H., Nasseri, A., Stahl, O., \& Zinnecker, H. 2012, MNRAS, 424, 1925

Claudi, R. U., Turatto, M., Gratton, R. G., et al. 2008, Proc. SPIE, 7014, 70143E

Cushing, M. C., Rayner, J. T., \& Vacca, W. D. 2005, ApJ, 623, 1115

Cushing, M. C., Marley, M. S., Saumon, D., et al. 2008, ApJ, 678, 1372

Cutri, R. M., Skrutskie, M. F., van Dyk, S., et al. 2003, 2MASS All Sky Catalog of Point Sources

Dent, W. R. F., Wyatt, M. C., Roberge, A., et al. 2014, Science, 343, 1490

de Zeeuw, P. T., Hoogerwerf, R., de Bruijne, J. H. J., Brown, A. G. A., \& Blaauw, A. $1999, \mathrm{AJ}, 117,354$

Dohlen, K., Langlois, M., Saisse, M., et al. 2008, Proc. SPIE, 7014, 70143L

Draine, B. T. 2003, ARA\&A, 41, 241

Faherty, J. K., Burgasser, A. J., Walter, F. M., et al. 2012, ApJ, 752, 56

Filippazzo, J. C., Rice, E. L., Faherty, J., et al. 2015, ApJ, 810, 158

Foreman-Mackey, D., Hogg, D. W., Lang, D., \& Goodman, J. 2013, PASP, 125 306

Gagné, J., Faherty, J. K., Cruz, K., et al. 2014a, ApJ, 785, L14

Gagné, J., Lafrenière, D., Doyon, R., et al. 2014b, ApJ, 792, L17

Gagné, J., Burgasser, A. J., Faherty, J. K., et al. 2015a, ApJ, 808, L20

Gagné, J., Faherty, J. K., Cruz, K. L., et al. 2015b, ApJS, 219, 33

Gagné, J., Mamajek, E. E., Malo, L., et al. 2018, ApJ, 856, 23

Grether, D., \& Lineweaver, C. H. 2006, ApJ, 640, 1051

Hagelberg, J., Ségransan, D., Udry, S., \& Wildi, F. 2016, MNRAS, 455, 2178

Hinkley, S., Pueyo, L., Faherty, J. K., et al. 2013, ApJ, 779, 153

Høg, E., Fabricius, C., Makarov, V. V., et al. 2000, A\&A, 355, L27

Houk, N. 1993, VizieR Online Data Catalog: III/51B

Janson, M., Bonavita, M., Klahr, H., \& Lafrenière, D. 2012, ApJ, 745, 4

Janson, M., Lafrenière, D., Jayawardhana, R., et al. 2013, ApJ, 773, 170

Johnson, J. A., Aller, K. M., Howard, A. W., \& Crepp, J. R. 2010, PASP, 122 905

Jones, J., White, R. J., Quinn, S., et al. 2016, ApJ, 822, L3

Jones, M. I., Jenkins, J. S., Bluhm, P., Rojo, P., \& Melo, C. H. F. 2014, A\&A, 566, A113

Kiraga, M. 2012, Acta Astron., 62, 67

Kraus, A. L., Ireland, M. J., Martinache, F., \& Lloyd, J. P. 2008, ApJ, 679, 762

Lachapelle, F.-R., Lafrenière, D., Gagné, J., et al. 2015, ApJ, 802, 61

Lafrenière, D., Jayawardhana, R., \& van Kerkwijk M. H. 2008, ApJ, 689, L153

Lafrenière, D., Jayawardhana, R., Janson, M., et al. 2011, ApJ, 730, 42

Lagrange, A.-M., Bonnefoy, M., Chauvin, G., et al. 2010, Science, 329, 57

Lagrange, A.-M., Langlois, M., Gratton, R., et al. 2016, A\&A, 586, L8

Lannier, J., Delorme, P., Lagrange, A. M., et al. 2016, A\&A, 596, A83

Leggett, S. K., Allard, F., Dahn, C., et al. 2000, ApJ, 535, 965

Liu, M. C., Dupuy, T. J., \& Allers, K. N. 2013, Astron. Nachr., 334, 85

Lodieu, N., Hambly, N. C., Jameson, R. F., \& Hodgkin, S. T. 2008, MNRAS, 383,1385

Lowrance, P. J., Schneider, G., Kirkpatrick, J. D., et al. 2000, ApJ, 541, 390

Luhman, K. L., Patten, B. M., Marengo, M., et al. 2007, ApJ, 654, 570

Macintosh, B. A., Graham, J. R., Palmer, D. W., et al. 2008, Proc. SPIE, 7015 18

Madsen, S., Dravins, D., \& Lindegren, L. 2002, A\&A, 381, 446

Maire, A.-L., Bonnefoy, M., Ginski, C., et al. 2016a, A\&A, 587, A56

Maire, A.-L., Langlois, M., Dohlen, K., et al. 2016b, Proc. SPIE, 9908, 990834

Marois, C., Lafrenière, D., Doyon, R., Macintosh, B., \& Nadeau, D. 2006, ApJ, 641,556

Marois, C., Correia, C., Véran, J.-P., \& Currie, T. 2014, IAU Symp., 299, 48

Mawet, D., David, T., Bottom, M., et al. 2015, ApJ, 811, 103

McLean, I. S., McGovern, M. R., Burgasser, A. J., et al. 2003, ApJ, 596, 561

Mesa, D., Gratton, R., Zurlo, A., et al. 2015, A\&A, 576, A121

Metchev, S. A., \& Hillenbrand, L. A. 2009, ApJS, 181, 62

Nielsen, E. L., Liu, M. C., Wahhaj, Z., et al. 2012, ApJ, 750, 53

Olofsson, J., Samland, M., Avenhaus, H., et al. 2016, A\&A, 591, A108

Patience, J., King, R. R., de Rosa, R. J., \& Marois, C. 2010, A\&A, 517, A76

Pavlov, A., Möller-Nilsson, O., Feldt, M., et al. 2008, Proc. SPIE, 7019, 701939

Pecaut, M. J., \& Mamajek, E. E. 2013, ApJS, 208, 9

Pecaut, M. J., \& Mamajek, E. E. 2016, MNRAS, 461, 794

Rayner, J. T., Cushing, M. C., \& Vacca, W. D. 2009, ApJS, 185, 289

Rizzuto, A. C., Ireland, M. J., \& Robertson, J. G. 2011, MNRAS, 416, 3108

Robert, J., Gagné, J., Artigau, É., et al. 2016, ApJ, 830, 144

Skrutskie, M., Cutri, R., Stiening, R., et al. 2006, AJ, 131, 1163
Slawson, R. W., Hill, R. J., \& Landstreet, J. D. 1992, ApJS, 82, 117 Soummer, R., Pueyo, L., \& Larkin, J. 2012, ApJ, 755, L28

Toomre, A. 1964, ApJ, 139, 1217

Torres, C. A. O., Quast, G. R., da Silva, L., et al. 2006, A\&A, 460, 695 van Leeuwen, F. 2007, A\&A, 474, 653

Vigan, A. 2016, Astrophysics Source Code Library [record ascl: 1603.001] Vigan, A., Langlois, M., Moutou, C., \& Dohlen, K. 2008, A\&A, 489, 1345

Vigan, A., Moutou, C., Langlois, M., et al. 2010, MNRAS, 407, 71

Vigan, A., Patience, J., Marois, C., et al. 2012, A\&A, 544, A9

Vigan, A., N'Diaye, M., Dohlen, K., et al. 2016, Proc. SPIE, 9912, 991226

Wright, E. L., Eisenhardt, P. R. M., Mainzer, A. K., et al. 2010, AJ, 140, 1868

Zurlo, A., Vigan, A., Galicher, R., et al. 2016, A\&A, 587, A57

1 Département d'Astronomie, Université de Genève, 51 chemin des Maillettes, 1290 Versoix, Switzerland e-mail: anthony . cheetham@unige.ch

2 Université Grenoble Alpes, CNRS, IPAG, 38000 Grenoble, France

3 INAF - Osservatorio Astronomico di Padova, Vicolo dell' Osservatorio 5, 35122 Padova, Italy

4 CRAL, UMR 5574, CNRS, Université de Lyon, Ecole Normale Supérieure de Lyon, 46 Allée d'Italie, 69364 Lyon Cedex 07, France

5 Aix-Marseille Université, CNRS, LAM (Laboratoire d'Astrophysique de Marseille) UMR 7326, 13388 Marseille, France

6 LESIA, Observatoire de Paris, PSL Research University, CNRS, Sorbonne Universités, UPMC Univ. Paris 06, Univ. Paris Diderot, Sorbonne Paris Cité, 5 place Jules Janssen, 92195 Meudon, France

7 Max Planck Institute for Astronomy, Königstuhl 17, 69117 Heidelberg, Germany

8 Unidad Mixta Internacional Franco-Chilena de Astronomía, CNRS/INSU UMI 3386 and Departamento de Astronomía, Universidad de Chile, Casilla 36-D, Santiago, Chile

9 Núcleo Milenio Formación Planetaria - NPF, Universidad de Valparaíso, Av. Gran Bretaña 1111, Valparaíso, Chile

10 Department of Astronomy, Stockholm University, AlbaNova University Center, 10691 Stockholm, Sweden

11 Institute for Astronomy, University of Edinburgh, Blackford Hill View, Edinburgh EH9 3HJ, UK

12 INCT, Universidad De Atacama, C/ Copayapu 485, Copiapó, Atacama, Chile

13 Institute of Astronomy, University of Cambridge, Madingley Road, Cambridge, CB3 0HA, UK

14 Exoplanets and Stellar Astrophysics Laboratory, Code 667, NASA Goddard Space Flight Center, Greenbelt MD, USA

15 Physikalisches Institut, University of Bern, Sidlerstrasse 5, 3012 Bern, Switzerland

16 Núcleo de Astronomía, Facultad de Ingeniería y Ciencias, Universidad Diego Portales, Av. Ejercito 441, Santiago, Chile

17 Institute for Particle Physics and Astrophysics, ETH Zurich, Wolfgang-Pauli-Strasse 27, 8093 Zurich, Switzerland

18 The University of Michigan, Ann Arbor, MI 48109, USA

19 School of Earth \& Space Exploration, Arizona State University, Tempe, AZ 85287, USA

20 Ural Federal University, Yekaterinburg 620002, Russia

21 Anton Pannekoek Institute for Astronomy, Science Park 904, 1098 XH Amsterdam, The Netherlands

22 Université Cote d'Azur, OCA, CNRS, Lagrange, France

23 INAF - Osservatorio Astrofisico di Arcetri, Largo E. Fermi 5, 50125 Firenze, Italy

24 INAF - Osservatorio Astronomico di Capodimonte, Salita Moiariello 16, 80131 Napoli, Italy

25 ONERA (Office National d'Etudes et de Recherches Aérospatiales), BP 72, 92322 Chatillon, France

26 European Southern Observatory (ESO), Karl-Schwarzschild-Str. 2, 85748 Garching, Germany

27 NOVA Optical Infrared Instrumentation Group, Oude Hoogeveensedijk 4, 7991 PD Dwingeloo, The Netherlands

28 European Southern Observatory (ESO), Alonso de Córdova 3107, Vitacura, Casilla 19001, Santiago, Chile 


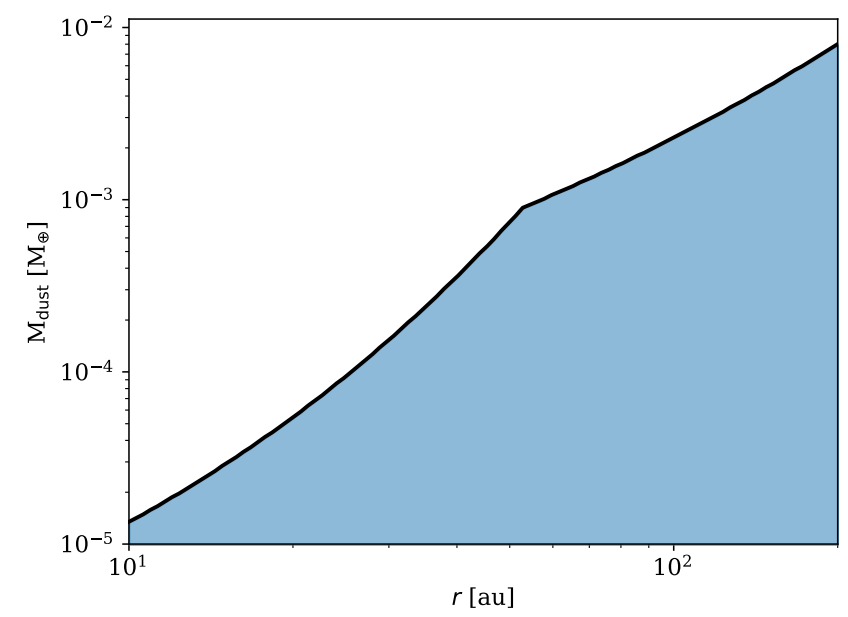

Fig. A.1. Blue-shaded area shows the region in the $r-M_{\text {dust }}$ plane, in which a debris disk could be present and remain compatible with the mid- and far-IR observations.

\section{Appendix A: Upper limits on the dust mass}

Chen et al. (2012) reported a non-detection of a mid-IR excess around HIP 64892, with a Spitzer/MIPS upper limit at $70 \mu \mathrm{m}$ of $15.8 \mathrm{mJy}$. Using a similar approach to that of Chauvin et al. (2017) for HIP 65426, we can convert this value to an upper limit on the dust mass around HIP 64892. Similar to HIP 65426, this upper limit does not reach the photospheric flux ( 1.4 mJy).

We gather the optical to mid-IR photometry of the star using the $\operatorname{VOSA}^{4}$ tool (Bayo et al. 2008). For the given stellar luminosity and mass ( $33 L_{\odot}$ and $2.35 M_{\odot}$, respectively) we estimate the size of dust grains that would still be on bound orbits around the star. We use the optical constant of astro-silicates (Draine 2003) and we compute the radiation pressure to gravitational forces $\beta$ ratio as in Burns et al. (1979). We find that for this composition, grains larger than $s_{\text {blow }} \sim 4.8 \mu \mathrm{m}$ should remain on bound orbits around the star. To estimate the possible configurations for a debris disk to remain compatible with the mid- and far-IR observations, we compute a series of disk models (similar to Olofsson et al. 2016). We consider a grain size distribution of the form $\mathrm{d} n(s) \propto s^{-3.5} \mathrm{~d} s$, between $s_{\text {min }}=s_{\text {blow }}$ and $s_{\max }=1 \mathrm{~mm}$. We sample $100 r_{\mathrm{i}}$ values for the radial distance of the belt between 10 and $200 \mathrm{au}$. For each $r_{\mathrm{i}}$, we consider a disk model between $0.9 \times r_{\mathrm{i}} \leq r_{\mathrm{i}} \leq 1.1 \times r_{\mathrm{i}}$. We then slowly increase the mass of the disk until the thermal emission (plus the stellar contribution) is larger than either the WISE/W4 $22 \mu \mathrm{m}$ point or the Spitzer/MIPS $70 \mu \mathrm{m}$ point. We therefore delimit a region in the $r$ $M_{\text {dust }}$ plane where debris disks could exist and remain undetected with the current observations (see Fig. A.1). Overall, with our assumptions on the radial extent of the debris disk, we find the dust mass must be less than $\sim 2 \times 10^{-3} M_{\oplus}$ at about 100 au from the star. When compared to the well-known $\beta$ Pictoris debris disk, we find that any potential belts around HIP 64892 must be substantially less massive. The total dust mass of the $\beta$ Pictoris debris disk was measured at $\sim 8 \times 10^{-2} M_{\oplus}$ (Dent et al. 2014) between 50 and $120 \mathrm{AU}$, implying a value of $\sim 3 \times 10^{-2} M_{\oplus}$ for an annulus at $100 \mathrm{AU}$ that can be compared with our simulation (assuming constant density).

\footnotetext{
4 http://svo2.cab.inta-csic.es/theory/vosa/
}

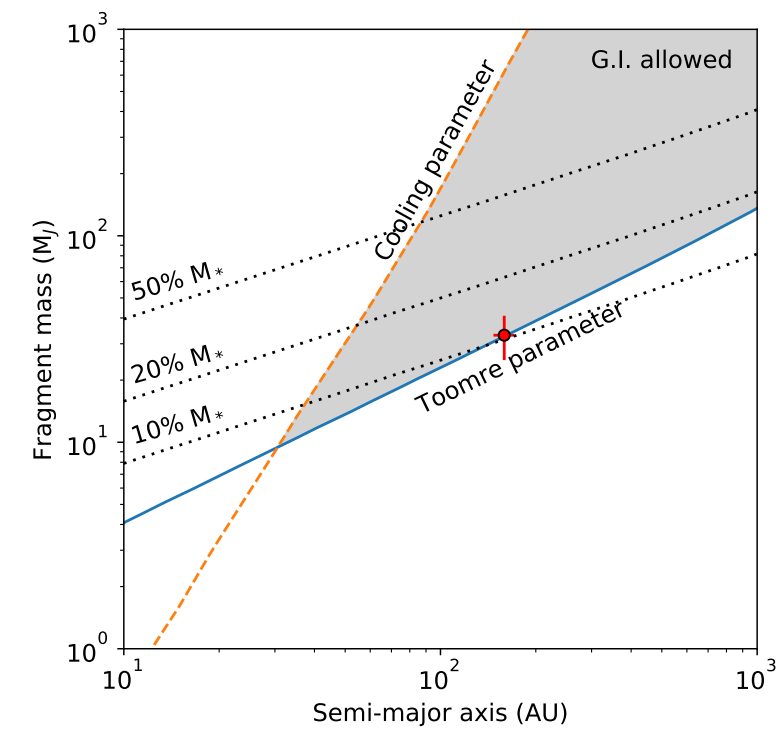

Fig. A.2. Predictions for the fragment masses compatible with production via gravitational instability for HIP 64892. Fragments with masses above the orange dashed line cannot cool efficiently enough, while those below the blue solid line do not satisfy the Toomre criterion. The mass and projected separation of HIP 64892B are marked. We find that HIP 64892B is compatible with in situ formation via gravitational instability, although further constraints on the semi-major axis are needed to confirm this idea. The initial disk masses required to form fragments of a given size are shown with black dotted lines.

\section{Appendix B: Disk instability models for HIP 64892B}

To investigate the possibility that HIP 64892B formed via disk instability, we estimate the range of fragment masses that could be produced as a function of semi-major axis using a set of fragmentation criteria as recently confirmed in local high-resolution three-dimensional simulations (Baehr et al. 2017). These models are described in detail in Janson et al. (2012) and Bonnefoy et al. (2014b). Briefly, fragments must satisfy the Toomre criterion for self-gravitating clumps (Toomre 1964) and be able to cool faster than the local Keplerian timescale. These models require the star's initial luminosity and metallicity. The former was estimated for HIP 64892 from the isochrones of Bressan et al. (2012), while for the latter we assumed a solar metallicity. The result is plotted in Fig. A.2. We also compared the primordial disk mass required to support fragments of a given mass for disks with $10 \%, 20 \%$, and $50 \%$ of the mass of the host star.

We found that if the currently observed projected separation of HIP 64892B is close to its semi-major axis, then its predicted mass and location are close to the Toomre limit. This opens the possibility that the companion may have formed in situ via gravitational instability.

The same models were applied by Bonnefoy et al. (2014b) to the companions $\kappa$ And B, HR 7329B, HD 1160B, and HIP $78530 \mathrm{~B}$. These objects are all young substellar companions to 2-2.5 $M_{\odot}$ stars. The brown dwarf HIP 64892B falls in a similar region of the diagram to $\kappa$ And B, HR 7329B, and HD 1160B. These four objects are compatible with in situ formation via disk instability and require only modest disk masses of 10-20\% of the mass of the primary star to form. The brown dwarf HIP 78530B is a clear outlier at a much larger separation that puts it below the Toomre limit, indicating that its formation proceeded through a different pathway, or that it underwent significant outward orbital migration following its formation epoch. 